\title{
Optical Sectioning of differential interference contrast microscopy
}

\author{
Atsushi Noguchi ${ }^{*}$, Hiroshi Ishiwata ${ }^{*}$, Masahide Itoh ${ }^{*}$ and Toyohiko Yatagai ${ }^{* * * *}$ \\ * Pure and Applied Sciences, University of Tsukuba, 1-1-1 Tennoudai, Tsukuba, Ibaraki \\ 305-8573, Japan
}

** Utsunomiya University Center for Optical Research \& Education(CORE), 7-1-2 Yoto, Utsunomiya, Tochigi 321-8585, Japan

E-mail:a.noguchi@sugino.com

\begin{abstract}
While developing a three dimensional measurement technique by the retardation-modulated differential interference contrast (RM-DIC) microscope, we found a problem. The problem is that the measurement range is restrict in $\lambda$ because it applies weak phase approximation.

To overcome this drawback, we propose a three dimensional (3-D) reconstruction method with z-axis scanning. This technique needs high optical sectioning like confocal microscopy.

We investigate the characteristic of optical sectioning in DIC microscope, then, we confirm that DIC microscope has high optical sectioning, experimentally. We also confirm that RM-DIC microscope has higher one.

Combining the optical sectioning of RM-DIC microscope and z-scanning, we develop a new 3-D reconstruction method. This novel technique overcomes the problem, the measurement range goes micron order.
\end{abstract}

\section{Introduction}

Differential interference contrast (DIC) microscopy utilizes interference between two polarized lights that passes through slightly different areas (amount of the shear: $\Delta$ ) of a specimen. It visualizes the optical path difference between the lights as differential [1].

DIC microscopy has big advantages in revealing detailed structures of unstained living cells and also in observing small steps on the surfaces of semiconductor wafers. It is very attractive microscopy for medical and biological research. Furthermore DIC microscope has high sensitivity to detect the sample information and high horizontal resolution, and it is thought that DIC microscope has strong optical sectioning.[15]

Previous researchers have tried and have shown the surface slope [2-4] or qualitative 
microstructure measurements [5-7]. Although these methods are useful, they cannot be extended to quantitative reconstruction of the microstructures of specimen.

We have developed retardation-modulated DIC (RM-DIC) microscope which could consider the diffracted light by microstructures of a sample. We have worked out the quantitative measurement in focal depth $[8,14]$.

RM-DIC microscope is expected to be a useful three-dimensional (3-D) measurement instruments in various field.

However, when the phase distributions of sample beyond the weak phase approximation range in quantitative measurement with RM-DIC microscope, we obtain smaller measurement values than the real phase distribution of a phase object. Especially, if the phase distribution goes over $\lambda / 4$ in reflection mode, the measurement result becomes upside down. As a result, quantitative measurement by RM-DIC microscope is restricted in $\lambda / 4$.

We have been investigating to extend the measurement range. To measure the phase distribution which smaller than $\lambda / 4$ accurately, we have developed the self phase correction (SPC) method[14]. To measure the depth larger than $\lambda / 4$, we have proposed the multi-wavelength method[11]. As a result, we extend the measurement range from $\lambda / 4$ to $\lambda$.

However, most samples are larger than the focal depth. To practical use, it is necessary to extend the measurement range much more.

The ordinary 3-D measurement by a microscopy, which has strong optical sectioning like confocal microscope, combines optical sectioning techniques with z-scanning (along optical axis).

Since it is thought that DIC microscope also has strong optical sectioning, we think that we can apply the same techniques to DIC microscope.

First, we explain the image characteristics in DIC system and 3-D reconstruction procedure. Second, we explain the characteristics of optical sectioning in DIC and RM-DIC microscopes theoretically. Third, we describe the new method of extending measurement range and show the results. Finally, we discuss a relationship between the shear amount and the optical sectioning effect and confirm it theoretically and experimentally.

\section{Theory}

\section{2-1. RM-DIC microscope}

In the beginning, we introduce the image characteristics of DIC microscope and the principle of quantitative 3-D measurement by RM-DIC microscope.

To simplify the description, we use a simple model that has a one-dimensional phase distribution along the $\mathrm{x}$-axis with constant phase along y-axis. We discuss the intensity distribution of a microscope image in the case of a pure phase object. We assume that the phase distribution of the object to be the function $\phi(x)$ with uniform reflectivity $C$. 
According to weak phase approximation, the object $o(x, y)$ and its Fourier transform $O\left(f_{x}, f_{y}\right)$ are expressed as

$$
o(x)=C \exp (i \phi(x))=C\left[1+i \phi(x)-\frac{1}{2} \phi(x)^{2}\right]
$$

and

$$
O\left(f_{x}\right)=C\left[\delta\left(f_{x}\right)+i \Phi\left(f_{x}\right)-\frac{1}{2} \Phi\left(f_{x}\right) \otimes \Phi^{*}\left(f_{x}\right)\right] .
$$

Where the symbol $\otimes$ is the convolution operator.

Substituting Eq.(2) to the partial coherence theory, the image characteristics of DIC microscope is shown as

$$
\begin{aligned}
I(x, \theta)= & C\left[\frac{1}{2}(1-\cos \theta)\left\{M(0)-\int_{-\infty}^{\infty} \cos \left(\pi \Delta f_{x}\right) M\left(f_{x}\right) \Phi\left(f_{x}\right) \otimes \Phi^{*}\left(f_{x}\right) \exp \left(-2 i \pi f_{x} x\right) d f_{x}\right\}\right. \\
& +i \sin \theta \int_{-\infty}^{\infty} \sin \left(\pi \Delta f_{x}\right) M\left(f_{x}\right) \Phi\left(f_{x}\right) \exp \left(-2 i \pi f_{x} x\right) d f_{x} \\
& +\frac{1}{2} \int_{-\infty}^{\infty}\left\{1-\cos \left(2 \pi \Delta f_{x}+\theta\right)\right\} m_{0}\left(f_{x}\right) \Phi\left(f_{x}\right) \Phi^{*}\left(f_{x}\right) d f_{x}
\end{aligned}
$$

In which

$$
\begin{aligned}
& M\left(f_{x}\right)=\iint_{-\infty}^{\infty} Q(\xi, \varsigma) p\left(\xi+f_{x}, \varsigma\right) p^{*}(\xi, \varsigma) d \xi d \varsigma, \\
& m_{0}\left(f_{x}\right)=\iint_{-\infty}^{\infty} Q(\xi, \varsigma) p\left(\xi+f_{x}, \varsigma\right) p^{*}\left(\xi+f_{x}, \varsigma\right) d \xi d \varsigma, \\
& m_{d}\left(f_{x}\right)=\iint_{-\infty}^{\infty} Q(\xi, \varsigma) p\left(\xi+f_{x}, \varsigma\right) p^{*}\left(\xi-f_{x}, \varsigma\right) d \xi d \varsigma,
\end{aligned}
$$

where $\Delta$ is the shear amount of DIC microscope, and $\theta$ is the retardation between two shared polarized lights in the DIC microscope, $Q(\xi, \varsigma)$ is the intensity distribution at the pupil of the illuminating optical system, $p(\xi, \varsigma)$ is the pupil function of imaging system.

It is assumed that microstructures are on a flat plane and the phase distribution of the microstructures is small. In this case, we can neglect the third and fourth terms of Eq.(3), which allows us to approximate Eq.(3) as

$$
I(x, \theta)=C\left[\frac{1}{2}(1-\cos \theta) M(0)-i \sin \theta \int_{-\infty}^{\infty} \sin \left(\pi \Delta f_{x}\right) M\left(f_{x}\right) \Phi\left(f_{x}\right) \exp \left(-2 i \pi f_{x} x\right) d f_{x}\right] \text { (4) }
$$

The first term of Eq.(4) means the image component of the transmitted or reflected light, therefore the background component of DIC image. The second term means the linear image component which is the differential of the phase distribution of microstructure. In this paper, we call the second term the phase component. 
As we take two DIC images with different retardations $( \pm \theta)$ then subtract and add these two DIC images, we can obtain [8]

$$
\begin{aligned}
& I(x, \theta)-I(x,-\theta)=2 C i \sin \theta \int_{-\infty}^{\infty} \sin \left(\pi \Delta f_{x}\right) M\left(f_{x}\right) \Phi\left(f_{x}\right) \exp \left(-2 i \pi f_{x} x\right) d f_{x}, \\
& I(x, \theta)+I(x,-\theta)=C(1-\cos \theta) M(0) .
\end{aligned}
$$

To calculate the phase distribution, we normalize Eq.(5) by Eq.(6) and apply it to the deconvolution operator of the modulation transfer function(MTF), so we can write

$$
\phi(x)=\frac{1-\cos \theta}{2 i \sin \theta} \operatorname{Decon}\left[\frac{I(x, \theta)-I(x,-\theta)}{I(x, \theta)+I(x,-\theta)}\right],
$$

where Decon[ ] is the deconvolution function. From Eq.(7), we can measure the phase distribution of microstructure of sample[8].

\section{2-2. Optical sectioning of DIC microscope}

We have been investigating to extend the measurement range, and have developed the SPC method [14] and the multi-wavelength method [11]. As a result, we have extended the measurement range from $\lambda / 4$ to $\lambda$, however, it is necessary to extend the measurement range for practical use. The most popular technique to extend the measurement range is combining optical sectioning techniques with z-scanning of the sample.

In this section, we consider the optical sectioning of DIC microscope. When defocus $\delta z$ which is the distance value from focal point happens in DIC microscope observation, the wavefront aberration occurs.

The pupil function is written as

$$
p(\xi, \varsigma, \delta \mathrm{z})=\exp \left(i \alpha \cdot \delta \mathrm{z}\left(\xi^{2}+\zeta^{2}\right)+\beta\right) \text {. }
$$

Where $\alpha$ means the aberration coefficient and $\beta$ means arbitrary number.

So, we can rewrite Eq.(3) as

$$
\begin{aligned}
I(x, \delta \mathrm{z}, \theta)= & C\left[\frac{1}{2}(1-\cos \theta)\left\{M(0)-\int_{-\infty}^{\infty} \cos \left(\pi \Delta f_{x}\right) M\left(f_{x}, \delta \mathrm{z}\right) \Phi\left(f_{x}\right) \otimes \Phi^{*}\left(f_{x}\right) \exp \left(-2 i \pi f_{x} x\right) d f_{x}\right\}\right. \\
& +i \sin \theta \int_{-\infty}^{\infty} \sin \left(\pi \Delta f_{x}\right) M\left(f_{x}, \delta \mathrm{z}\right) \Phi\left(f_{x}\right) \exp \left(-2 i \pi f_{x} x\right) d f_{x} \\
& +\frac{1}{2} \int_{-\infty}^{\infty}\left\{1-\cos \left(2 \pi \Delta f_{x}+\theta\right)\right\} m_{0}\left(f_{x}, \delta \mathrm{z}\right) \Phi\left(f_{x}\right) \Phi^{*}\left(f_{x}\right) d f_{x} \\
& \left.+\frac{1}{2} \int_{-\infty}^{\infty}\left\{\cos \theta+\cos \left(2 \pi \Delta f_{x}\right)\right\} m_{d}\left(f_{x}, \delta \mathrm{z}\right) \Phi\left(f_{x}\right) \Phi^{*}\left(-f_{x}\right) \exp \left(-4 i \pi f_{x} x\right) d f_{x}\right], \\
M\left(f_{x}, \delta \mathrm{z}\right)= & \iint_{-\infty}^{\infty} Q(\xi, \varsigma) p\left(\xi+f_{x}, \varsigma, \delta \mathrm{z}\right) p^{*}(\xi, \varsigma, \delta \mathrm{z}) d \xi d \varsigma
\end{aligned}
$$


$m_{0}\left(f_{x}, \delta \mathrm{z}\right)=\iint_{-\infty}^{\infty} Q(\xi, \varsigma) p\left(\xi+f_{x}, \varsigma, \delta \mathrm{z}\right) p^{*}\left(\xi+f_{x}, \varsigma, \delta \mathrm{z}\right) d \xi d \varsigma$

$m_{d}\left(f_{x}, \delta \mathrm{z}\right)=\iint_{-\infty}^{\infty} Q(\xi, \varsigma) p\left(\xi+f_{x}, \varsigma, \delta \mathrm{z}\right) p^{*}\left(\xi-f_{x}, \varsigma, \delta \mathrm{z}\right) d \xi d \varsigma$

In Eq.(9), $m_{0}\left(f_{x}, \delta \mathrm{z}\right)$ in the third term is taking complex conjugate of pupil function with itself, and $m_{d}\left(f_{x}, \delta \mathrm{z}\right)$ in the fourth term is taking autocorrelation of pupil function with symmetry one.

Since the wavefront aberration of defocus has axial symmetry, we can assume that the influences of wavefront aberration to third term and fourth term are small. The wave front aberration of defocus effects to only $M\left(f_{x}, \delta \mathrm{z}\right)$ of the first term and the second term.

We shows the MTF $(M(f))$ of bright field microscope which is most popular microscope in Fig.1. When defocus happens, $M(f)$ drops around the intermediate frequency remarkably, but it hardly drops at low frequency region. In Eq.(9), the background component consists of a product of $\mathrm{M}(f)$ and $\cos \left(\pi \Delta f_{x}\right)$, so the image characteristics of background component is hardly changed by defocus.

On the other hand, the phase component multiplies $\mathrm{M}(f)$ by $\sin \left(\pi \Delta f_{x}\right)$. The sensitivity of intermediate frequency region becomes high and that of low frequency region become low. The image characteristic change by defocus is more remarkable than that of bright field microscope.

Consequently, DIC microscope has stronger optical sectioning effect than bright field microscope.

\section{2-3. Optical sectioning of RM-DIC microscope}

RM-DIC microscope can measure the phase component which is sensitive to defocus and the background component which is insensitive to defocus from DIC microscope images separately.

RM-DIC microscope can extract the changes of the phase component more sensitively than the conventional DIC microscope. Therefore, RM-DIC microscope has stronger optical sectioning than DIC microscope. We show the comparing the changes of bright field with that of DIC microscope and that of RM-DIC microscope in Fig.2.

When defocus happens, image of RM-DIC microscope changes most sensitive, and bright field microscope has the worst.

\section{2-4. Extending measurement range using optical sectioning of RM-DIC microscope}

In this section, we explain a new method to extend the measurement range of RM-DIC microscope. This new method utilizes optical sectioning of RM-DIC microscope.

First, we take several images of RM-DIC microscope as dataset with z-scanning. The dataset has 
positive and negative retardation ( $\pm \theta$ ). Secondly, we subtract these dataset as Eq.(5) and get rid of the back ground component. This operation extracts the pure phase component images. The extracted phase component has plus and minus components which corresponds to concavity and convexity. Thirdly, we take the absolute value of the extracted the phase component $I_{d i f}(x, z)$ as

$$
I_{d i f}(x, z)=|I(x, z, \theta)-I(x, z,-\theta)| .
$$

The phase component has the maximum value at focal area.

Finally, we compare the datasets at a certain point as

$$
I_{d i f}(x, 1)<\cdots \cdots I_{d i f}(x, z-1)<I_{d i f}(x, z)>I_{d i f}(x, z+1) \cdots \cdots I_{d i f}(x, Z) .
$$

Then, we set a threshold value and take follow operation

$$
\operatorname{mask}(x, z)=\left\{\begin{array}{ll}
0 & \left(I_{d i f}(x, z) \leq \text { threshold }\right) \\
1 & \left(I_{d i f}(x, z)>\text { threshold }\right)
\end{array}\right. \text {. }
$$

This binary image processing enables us to make a mask which cut out the infocus area. We call this mask as sectioning mask.

Combining the Sectioning mask which is made by the $\mathrm{Z}$ scan techniques and Eq.(7), we can extend the measurement range beyond the focal depth. We call this techniques focal phase reconstruction method (FPRM).

\section{2-5. Dependence on shear in optical sectioning of RM-DIC microscope}

As the phase component contains $\sin \left(\pi \Delta f_{x}\right)$ in the MTF, the MTF of phase component should depend on the shear amount. To confirm its dependence, we simulate the MTF of phase component (Fig.3). The result indicates that the MTF is not sensitive at low frequency region, especially no sensitivity at DC component and has band pass characteristics at intermediate frequency region. Furthermore, the smaller the shear amount become, the more sensitive the MTF become at high frequency. In fact, it is thought that there is a dependence on shear amount in optical sectioning of RM-DIC microscope.

\section{Experiments}

In this section, we explain three cases in experiments. We test the availability of the new measurement method, FPRM in case 1.

In the section 2-5, we explained a simulation result which showed that there was a dependence on shear in optical sectioning. We ascertain the dependence on shear in case 2.

Since the FPRM utilizes optical sectioning, we expect that there is a dependence on shear in the FPRM. We verify the dependence on shear in case 3. 


\section{3-1. Experimental setup}

We have developed RM-DIC microscope that includes a modulating retardation unit in the illuminating optics of a conventional DIC microscope (Olympus BX60 for DIC), and have applied the FPRM to this microscope. A block diagram of our setup is shown in Fig.4. We use Olympus Mplan100x for the objective lens. The light source is a halogen lamp. Using a interference filter, we can set center wavelength for observation at $550 \mathrm{~nm}$.

For case 1 and 3, We combine the stage of RM-DIC microscope with a PZT actuator (PI Co., $\mathrm{P}-915 \mathrm{~K} 019)$. The stage is controlled by a personal computer. The CCD camera is a $1 / 3$ " (B/W) VGA type and is connected to a frame grabber in the personal computer.

According to the experiment, we use three Nomarski prisms of different shear amounts ie $\Delta, \Delta / 2$, $\Delta / 3$.

\section{3-2, Samples}

For the three cases, we use three samples which are proper to each experiment.

A sample for case 1 has character patterns on a silicon wafer formed by a semiconductor process. The character patterns are covered by gold thin layer and $\mathrm{SiO} 2$. The depths of the character patterns are unknown.

A sample for case 2 is a coarsely polished glass plate. The rough surface is evaporated by gold and it has various spatial frequency components on the surface (Fig.5). The depths of these microstructures are around $10 \mathrm{~nm}$. DIC microscope can detect the microstructures easily.

A sample for case 3 is grating patterns on a silicon wafer which is made by a semiconductor process. The grating patterns have line widths from 0.5 to $3 \mathrm{um}$ and are covered with an aluminum layer. The depths of the grating patterns are $75 \mathrm{~nm}$.

\section{3-3. Measurement}

\section{Case 1 : Availability of the FPRM}

Using the first sample of which has character patterns, we take some datasets with RM-DIC microscope. Each dataset is taken every 100nm step along the optical axis controlled by PZT.

From the dataset, we make sectioning mask explained in section 2-4. We calculate the phase component from each images by Eq.(7). Combining the phase component with sectioning mask, we can extract the phase distribution within focal depth.

To test the availability of the FPRM, we compare the phase component reconstructed by the FPRM with by the white light interferometer (WLI).

\section{Case 2 : Dependence on shear in optical sectioning}

From the simulation result shown in Fig.3, it is thought that the MTF of the phase component, the 
second term of Eq.(4), extracted by RM-DIC microscope has the dependence on shear amount in the optical sectioning of RM-DIC microscope.

To confirm the dependence, we calculate the power spectrum from RM-DIC microscope image. Using the coarsely polished glass plate sample, we take datasets of focused images. The power spectrum is equivalent for the MTF of the phase component.

\section{Case 3 : Dependence on shear in the FPRM}

We think that the MTF of the phase component extracted by RM-DIC microscope has the dependence on shear amount in the optical sectioning of RM-DIC microscope. Accordingly, we can predict that the FPRM also has the dependence on shear amount. To practical use of the FPRM, we have to reveal the dependence.

Therefore, we make a sectioning mask of the grating sample inclining along the shear direction for a perpendicular to the groove for a few degrees. When the surface structures has not so large and not so deep, we can expect the sectioning mask techniques to make a belt-like sectioning mask orthogonally with the shear direction.

In this case, the relation is expressed as

$d=L \tan \theta$.

Where the symbol $d$ is the optical sectioning depth, $L$ is the sectioning mask width and $\theta$ is the inclining angle of sample. Since $d$ is proportional to $L$, we can assess optical sectioning depth from $L$ (Fig.6).

\section{Results and discussion}

First, we show the sectioning mask result of case 1 in Fig.7 It indicates that the focal area is extracted accurately. Using the same type sample, we can compare the measurement depth accuracy of the FPRM with the measurement of WLI shown in Fig.8. The measurement depth of the FPRM and WLI are $1.1 \mu \mathrm{m}$, and $1.2 \mu \mathrm{m}$ respectively. The result is quite similar.

Furthermore, since the FPRM uses RM-DIC microscope, the FPRM can reconstruct the microstructures which the WLI can't reconstruct.

We developed the FPRM which enables us to extend the measurement range and to measure the nanometer-order microstructures. This method can measure larger sample (like mm order height) which has microstructure in it.

Second, we show the result of case 2. Figure 9 shows power spectrum taken from the images of rough surface by RM-DIC microscope. The power spectrum curve is remarkably similar to the simulation result shown in Fig.3 except for the low frequency region including the DC component.

There are two exceptions at the low frequency region. One is a spike near the DC component. Let us consider why the spike is close to the DC component. We assumed that there is no background component by Eq.(8). But, actually there is a certain bias in the all image area (Fig.10). It means that 
the sample was inclined a little along the shear direction. Since this bias affects to the DC component, the MTF around DC component showed the spike shape. Another is a difference in the slope of MTF curve slopes at high frequency region. When we have calculated the simulation, we have assumed that the optical system was aberration-free. On the other hand, the measurement result by RM-DIC microscope includes the information from out of focus. These differences of condition when we calculated the MTF resulted in the MTF curve difference. Consequently, we confirmed that the smaller the shear amount become, the more sensitive the MTF become at high frequency.

Generally, optical sectioning depends on the image characteristics. In the case of RM-DIC microscope, the image characteristics depend on shear amount, so we found that optical sectioning of RM-DIC microscope depends on shear amount.

Finally, we describe the result of case 3. Figure 11 shows the sectioning mask. The results of shear amount $\Delta / 3$ tend to have low contrast than that of shear amount $\Delta$.

DIC microscope utilizes a spatial differential by shear amount. When shear amount is $\Delta$, the phase component per shear amount is larger than that of $\Delta / 3$ (Fig.12). Since the contrast is proportional to the phase component, the contrast is proportional to shear amount.

When the line width was $0.5 \mu \mathrm{m}$, the smaller the shear amount become, the thinner the sectioning mask width $L$ become. It means that the optical sectioning when shear amount was $\Delta$ is stronger than was $\Delta / 3$. Therefore, this result bears out the simulation except when the line width is larger than $2 \mu \mathrm{m}$.

To analyze how wide line width is suitable to observe by RM-DIC microscope, we calculated the spatial frequency of grating samples (Line width $0.5-3 \mu \mathrm{m}$ ) and plotted it with the power spectrum of RM-DIC microscope in Fig.9. The power spectrum and the spatial frequency in Fig.9 have sensitivity at cutoff frequency. We can consider the sensitivity as white noise. Since we normalized the MTF to understand the difference easily, the white noise on the MTF was emphasized.

When the line width was $2.0 \mu \mathrm{m}$ and above, the spatial frequency correspond to low frequency where the MTF was insensitive. Therefore, we couldn't make the sectioning mask properly. From this result, the DIC microscope is proper to observe an object which has microstructures like in living cell.

Consequently, for better use of FPRM, we should use a Nomarski prism which has suitable shear amount. For small object, Nomarski prism which has small shear amount like that of $\Delta / 3$ in case 3 is better.

\section{Conclusion}

We have been developing RM-DIC microscope and we succeeded quantitative measurements of phase distribution. To practical use, it was necessary to extend the measurement range. We analyzed optical sectioning of DIC microscope, and confirmed that the optical sectioning is strong because the 
MTF of phase component has a band pass characteristics. Especially, RM-DIC microscope can extract the phase component. We confirmed that RM-DIC microscope has stronger optical sectioning than that of a conventional DIC microscope.

To extend the measurement range, we developed the FPRM combined the strong optical sectioning of RM-DIC microscope with z-scanning. There was little difference between the FPRM and the WLI in the measurement depth. Furthermore, since the FPRM used RM-DIC microscope, the FPRM can reconstruct the microstructures than the WLI. The FPRM enable us to measure the $\mu \mathrm{m}$ order and above.

We found the dependence on shear in optical sectioning of RM-DIC microscope and analyzed the dependence on shear to the FPRM.

We think that the FPRM is expected to be a powerful tool for 3-D phase measurement.

\section{Reference}

1) M. Francon, Progress in Microscopy, Pergamon Press, New York (1961), p.118-119

2) D. L. Lessor, J. S. Hartman, R. L. Gordon, J. Opt. Soc., vol. 69(1979), pp 357-366

3) J. S. Hartman, R. L. Gordon, D. L. Lessor, Appl. Opt., vol. 19(1980), pp2998-3009

4) J. S. Hartman, R. L. Gordon, D. L. Lessor, Appl. Opt., vol. 20(1981) ,pp2665-2669

5) M. R. Arnison, C. J. Cogswell, N. I. Smith, P. W. Fekete, K. G. Larkin, J. of Microscopy, vol. 199 (2000), pp79-84

6) Shribak, S. Inoue, Appl. Opt., vol.45 (2006), pp460-469

7) Shribak, J. LaFountain, D. Biggs, S. Inoue, Proc. of SPIE, vol. 6441 (2007)

8) H. Ishiwata, M. Itoh, T. Yatagai, Opt. Commu., vol. 260 (2006), pp117-126

9) H. Hopkins, "The frequency response of a defocused optical system", Proc. Phys. Soc. 231(1955) , 91-103

10) A. R. FitzGerrell, E. R. Dowski Jr, W. T. Cathey, "Defocus transfer function for circularly symmetric pupils", Appl. Opt. 36 (1997), 5796-5804

11) K. Kondo, H. Ishiwata, M. Itoh, T. Yatagai, Technical Digest OIE (1999)

12) K. R. Castleman, Digital Image Processing, Prentice hall, (1996)

13) K. Itoh, A. Hayashi, Y. Ichioka, "Digitized optical microscopy with extended depth of field" Appl. Opt., vol. 28 (1989), pp3487-3493

14) H. Ishiwata, M. Itoh, T. Yatagai, "A new analysis for extending the measurement range of the retardation-modulated differential interference contrast (RM-DIC) microscope”, Opt. Commu., 281 (2008), 1412

15) S. Inoue and K. R. Spring, Videomicroscopy: The Fundamentals, 2nd ed. (Plenum, 1997) 


\section{Figure captions}

Figure 1 Modulation transfer function MTF $(\mathrm{M}(\mathrm{f}))$ of bright field microscope

$\mathrm{M}(\mathrm{f})$ drops around the intermediate frequency by defocus. However it hardly drops at low frequency region. In case of the phase component, it is multiplied $\mathrm{M}(f)$ by $\sin \left(\pi \Delta f_{x}\right)$. The sensitivity of intermediate frequency region becomes high and that of low frequency region become low.

Figure 2 Comparing the changes of image characteristics of bright field (BF) microscopes with DIC microscope and RM-DIC microscope. Image characteristics are changed by defocus.

In case of BF microscope, it is hardly changed by defocus. RM-DIC microscope has the most remarkable changes. It has stronger optical sectioning than DIC microscope.

Figure 3 Simulation of the MTF of differential component

As we have predicted, the MTF has no sensitivity at DC component. It has band pass characteristics at intermediate frequency region and has dependence on shear in MTF at high frequency region.

Figure 4 A block diagram of RM-DIC microscope.

In this paper, we use reflection type DIC microscope. LS is light source. IF is a interference filter.

PM is the Phase modulator. PZT is a piezo actuator. We use some Nomarski prisms of the three prisms, the shear amounts are $\Delta, \Delta / 2, \Delta / 3$.

Figure 5 the rough surface

We use a coarsely polished glass plate which is covered by gold as rough surface. There are various frequency components. The depths of these microstructures are $10 \mathrm{~nm}$ or so. DIC microscope can detect the microstructures positively.

Figure 6 The sample setup in Experiment 3

$d$ is optical sectioning depth, $L$ is the sectioning mask width and $\beta$ is the inclining angle. In this case, $d$ is proportional to $L$. So we can assess optical sectioning from the width $L$

Figure 7 the result of sectioning mask.

The defocus value is (a)+200nm,(b)0nm,(c)-200nm. Multiple the subtract image and the sectioning mask, we can extract the focal area accurately.

Figure.8.comparing with WLI

Reconstruct result by (a)FPRM and (b)WLI. We indicate the cross-section view below the result image. The measurement depth of the FPRM is $1.1 \mu \mathrm{m}$, the WLI is $1.2 \mu \mathrm{m}$. Since the FPRM uses RM-DIC microscope, the FPRM can reconstruct the microstructures.

Figure 9 The power spectrum taken from the images of rough surface by RM-DIC microscope

Dash lines are simulation results. Heavy lines are experimental results. Chain lines are spatial frequency of grating samples. The experimental curve is similar to that of simulation except a few 
points. From the spatial frequency of grating samples, we can confirm that a sample which has smaller line width than $1 \mu \mathrm{m}$ is proper to measure in our experimental setting.

Figure 10 A certain bias in the subtracted image

We assumed that there is no background component in subtracted image. But, actually there is a certain bias in the all image area. In frequency region, It means that the sample includes DC component.

Figure 11 Shows the sectioning mask

Shear amount (i) $\Delta$, (ii) $\Delta / 3$. The line width of grating sample (a) $0.5 u m$,(b) $1.2 u m$, (c) $2 u m$.

In case of the line width are $0.5 \mathrm{um}$ and $1.2 \mathrm{um}$, the sectioning mask width when shear is $\Delta / 3$ are thinner than that of $\Delta$. It means that the optical sectioning is strong. Therefore, this result bears out the simulation except when the line width is larger than $2 \mu \mathrm{m}$.

Figure 12 Relation between shear and the intensity.

(a) Ideal sample. Intensity models when the shear is (b) $\Delta$, (c) $\Delta / 3$. The phase component is proportional to the contrast. 


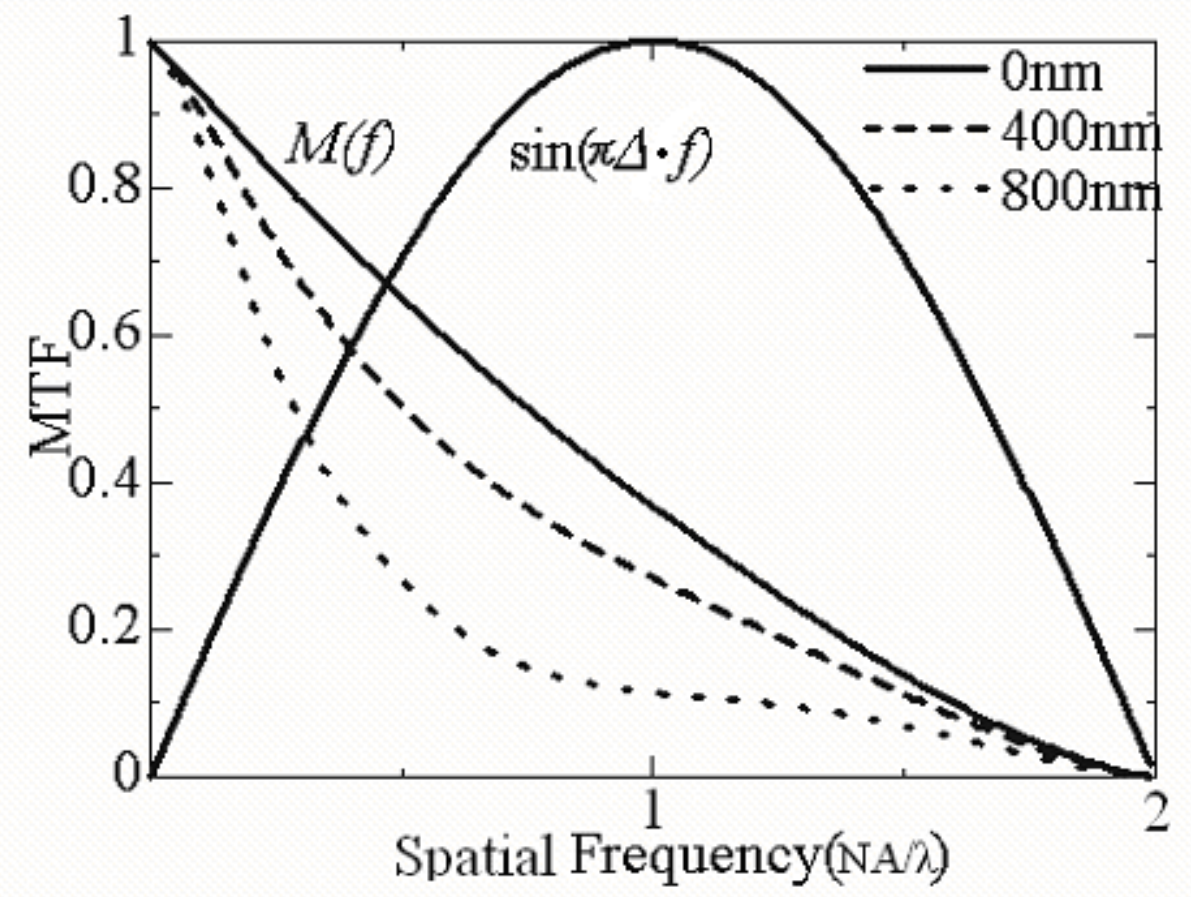

Figure 1 Modulation transfer function MTF (M(f)) of a bright field microscope 


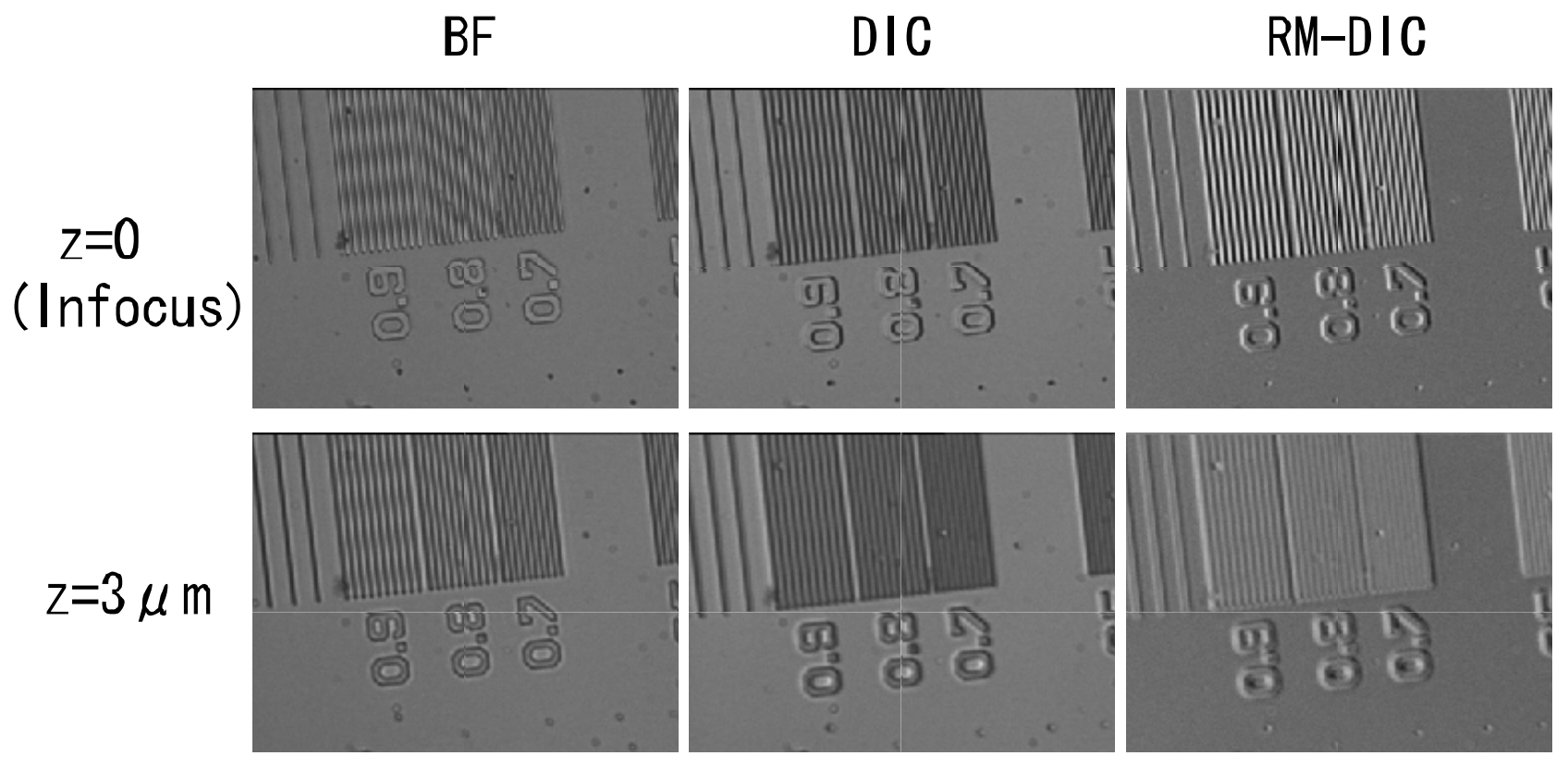

Figure 2 Comparing the changes of image characteristics of bright field (BF) microscopes with DIC microscope and RM-DIC microscope. 


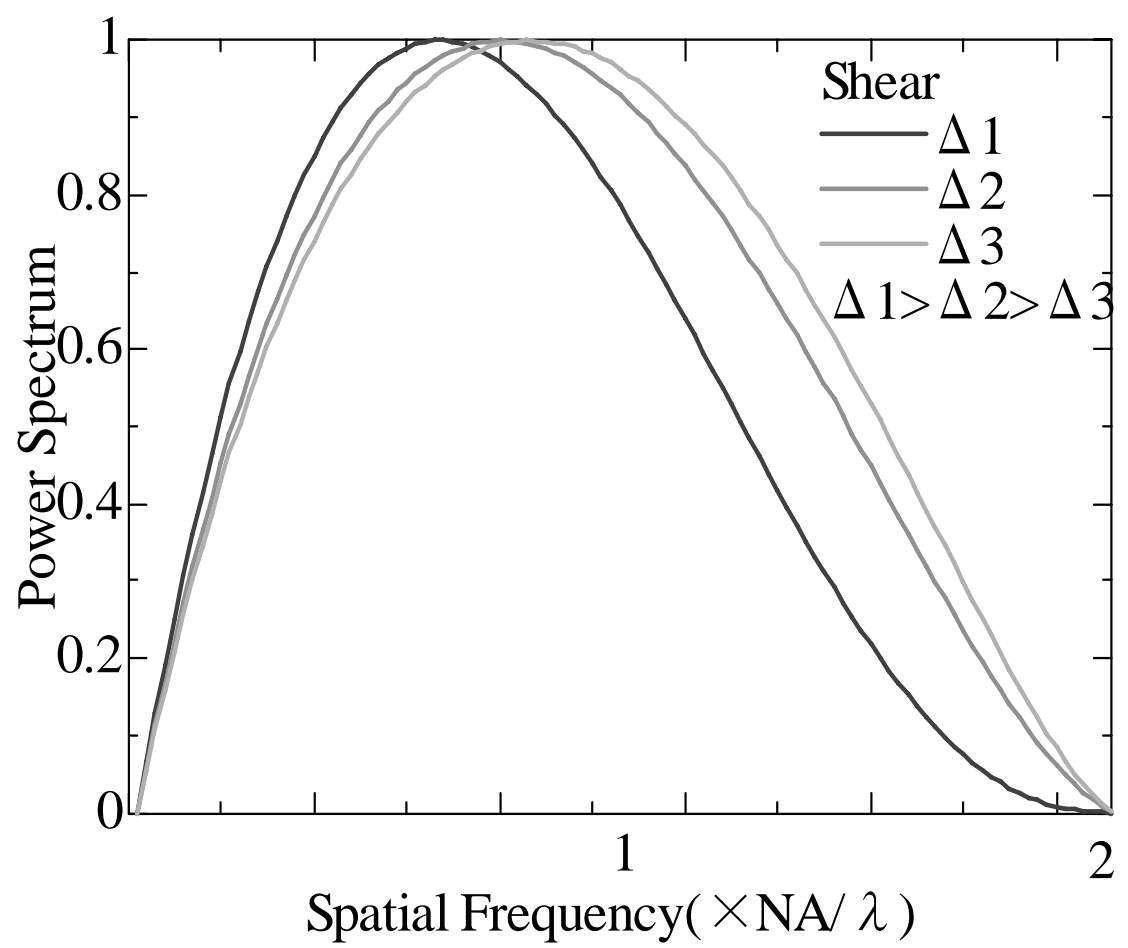

Figure 3 Simulation of the MTF of differential component 


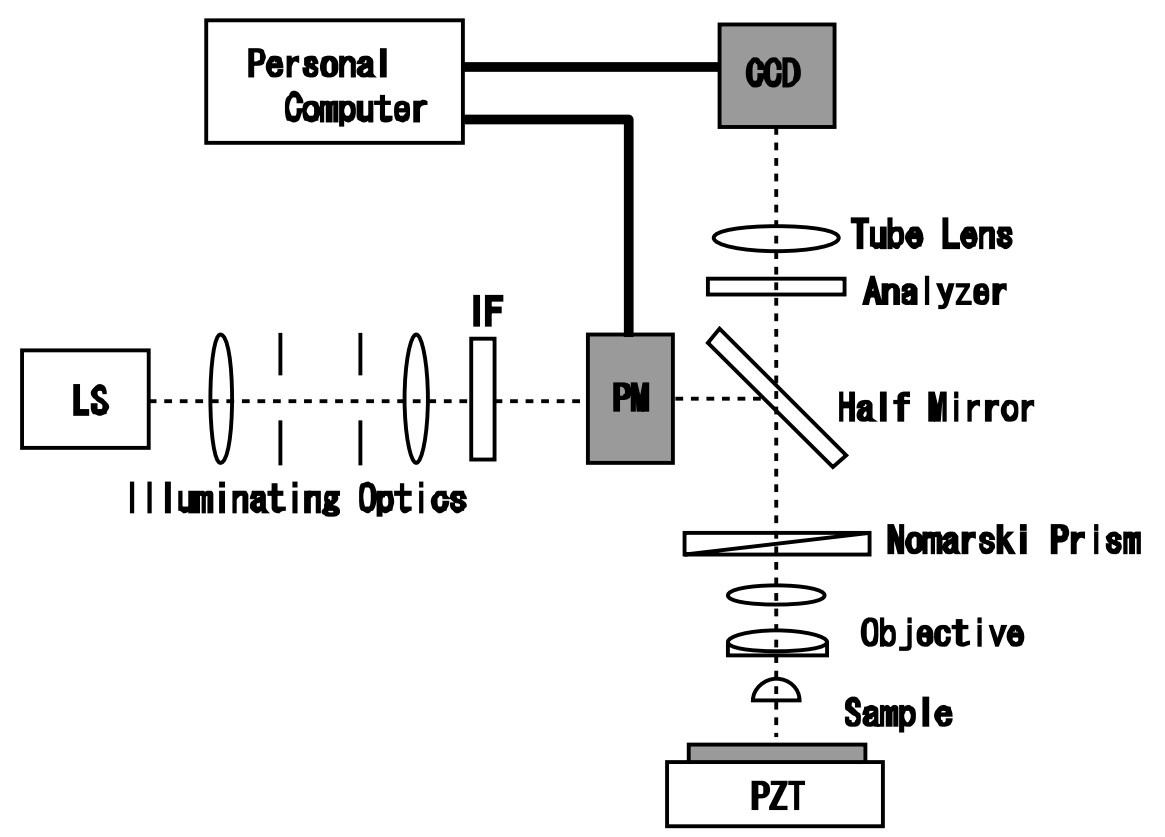

Figure 4 A block diagram of RM-DIC microscope. 


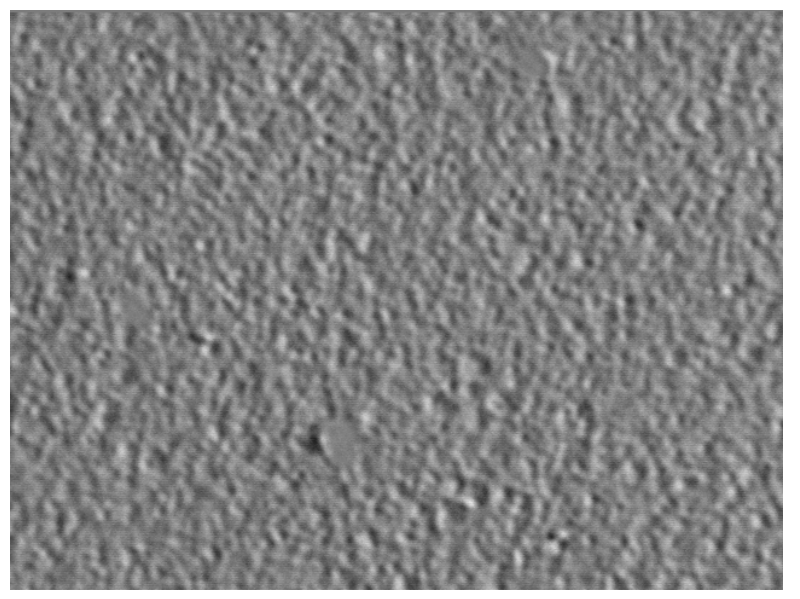

Figure 5 the rough surface 


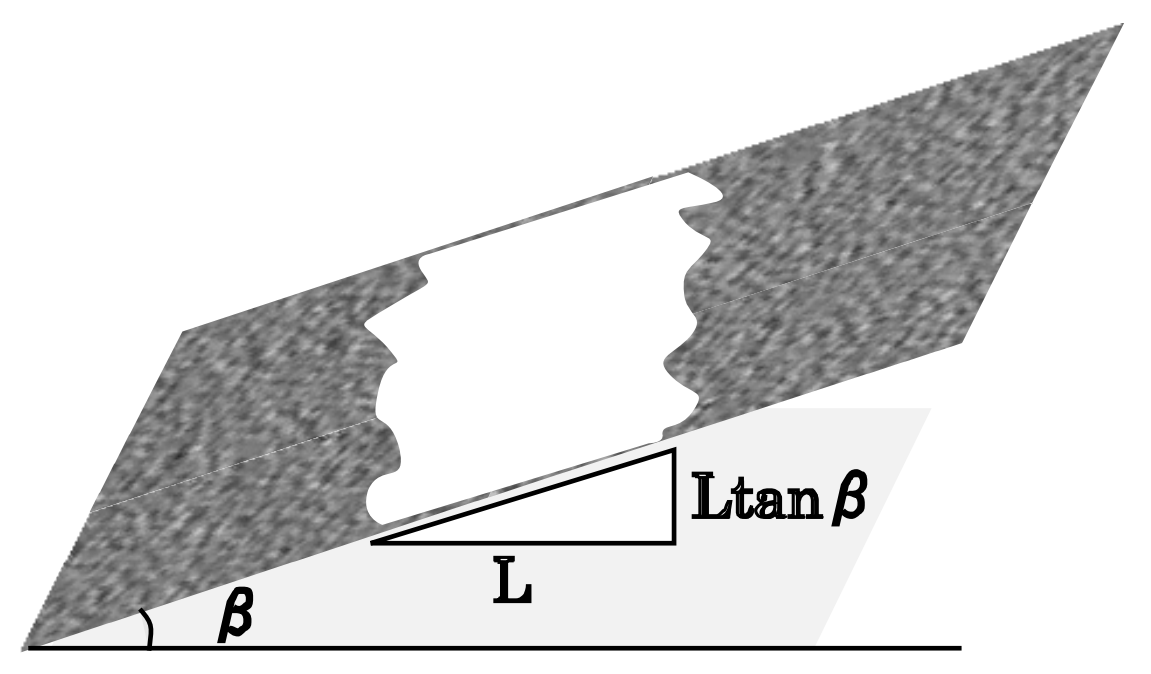

Figure 6 The sample setup in Experiment 3 
(a)

Subtructed image

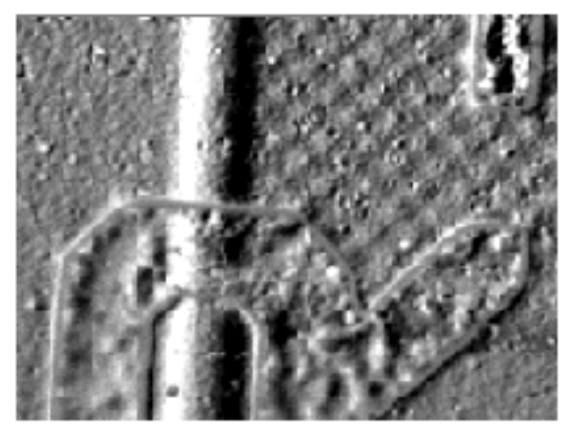

(b)
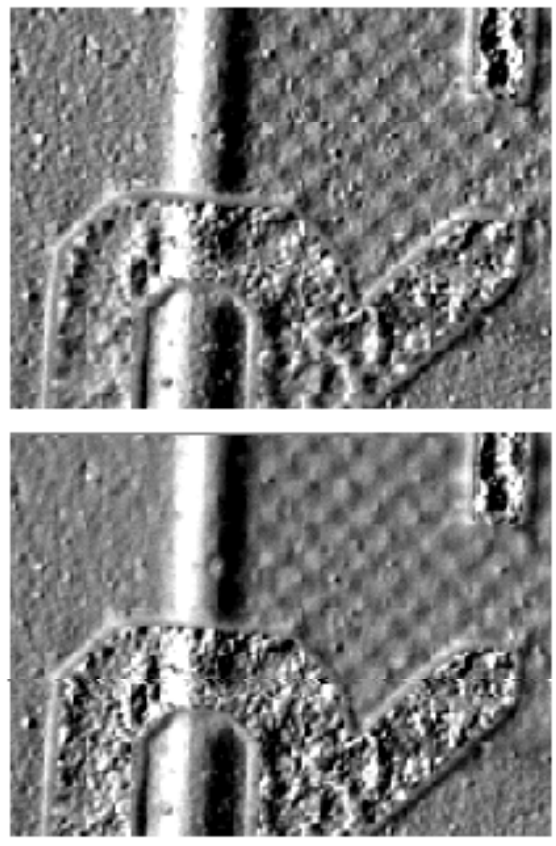

Sectioning mask
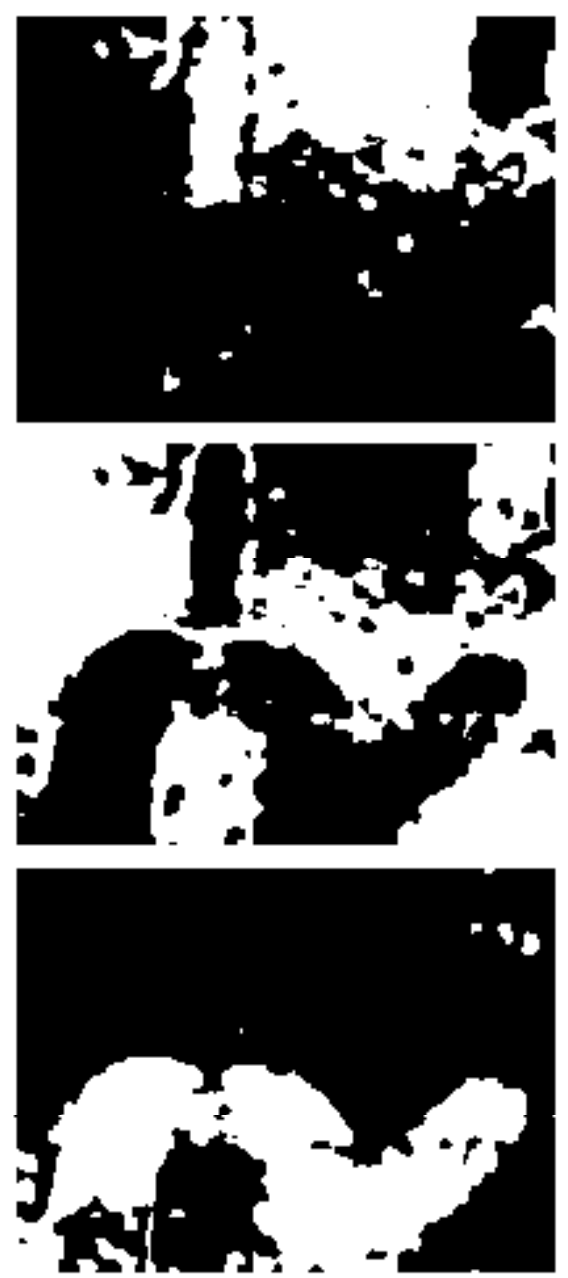

Extracted area
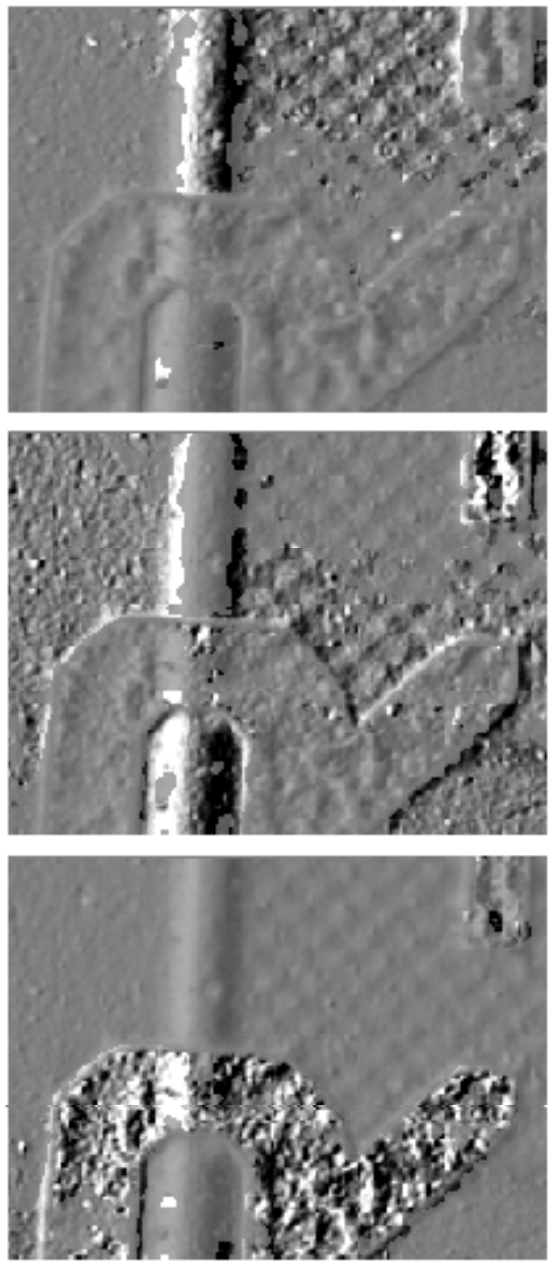

Figure 7 the result of sectioning mask. 
(a)
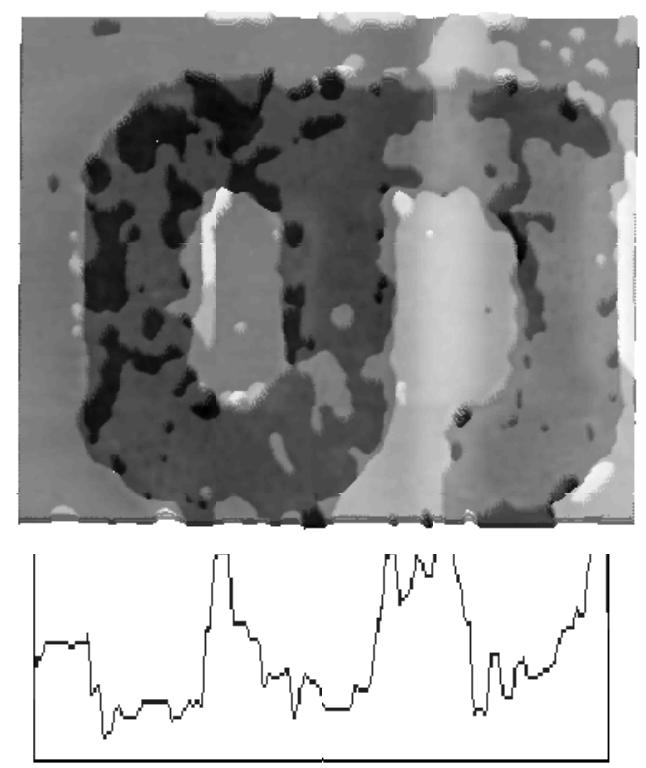

(b)

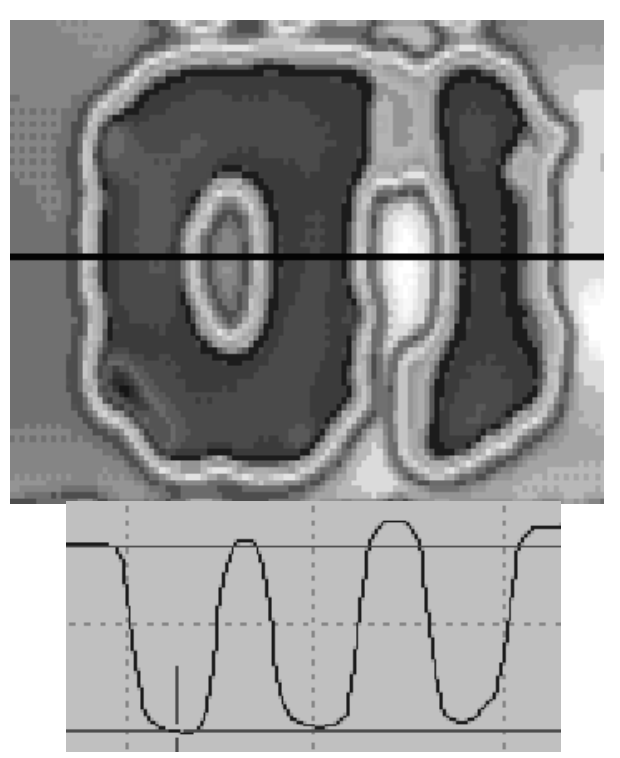

Figure.8.comparing with WLI 


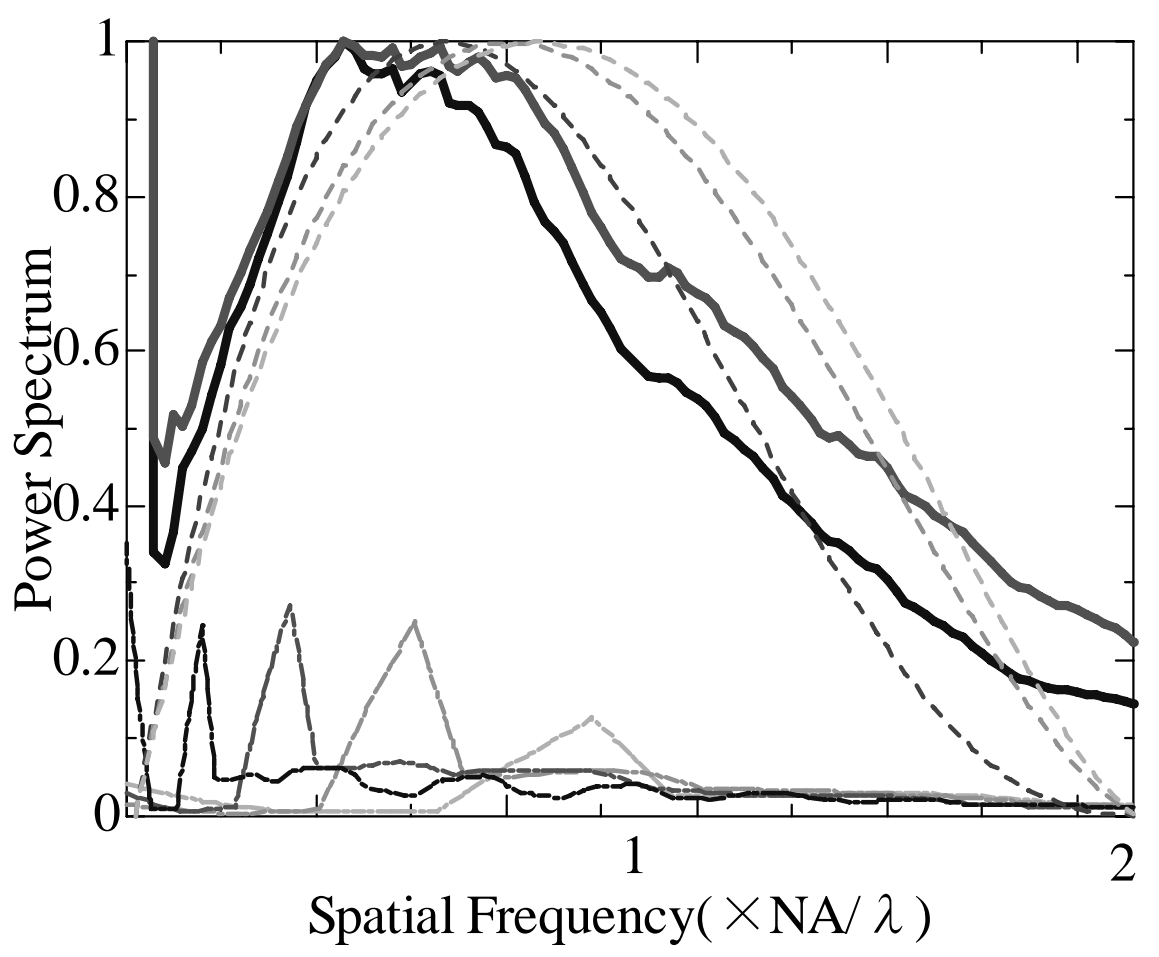

Shear(Experiment)

$-\Delta 1$

$-\Delta 3$

Shear(Simulation)

----- $\Delta 1$

$-\cdots-\Delta 2$

$---\Delta 3$

$\Delta 1>\Delta 2>\Delta 3$

LineWidth

$-0.5 \mu \mathrm{m}$

$0.8 \mu \mathrm{m}$

$-1.4 \mu \mathrm{m}$

$-3.0 \mu \mathrm{m}$

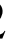

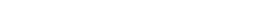




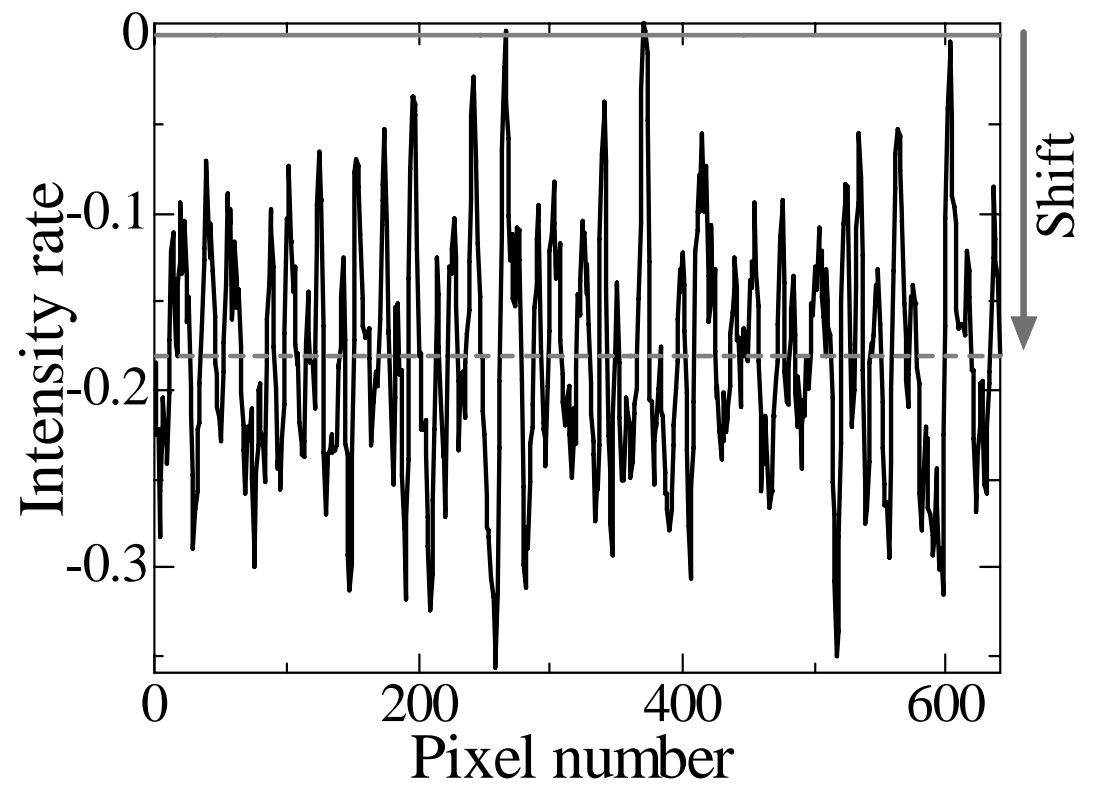

Figure $10 \mathrm{~A}$ certain bias in the subtracted image 
(i)

(ii )

Subtructed image Sectioning mask Subtructed image

Sectioning mask

(a)
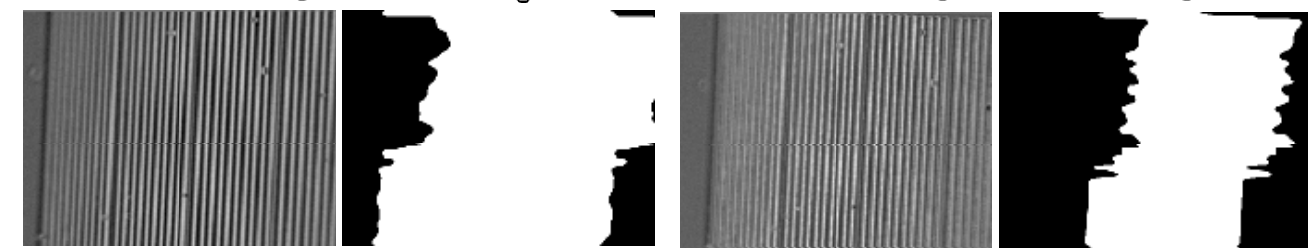

(b)
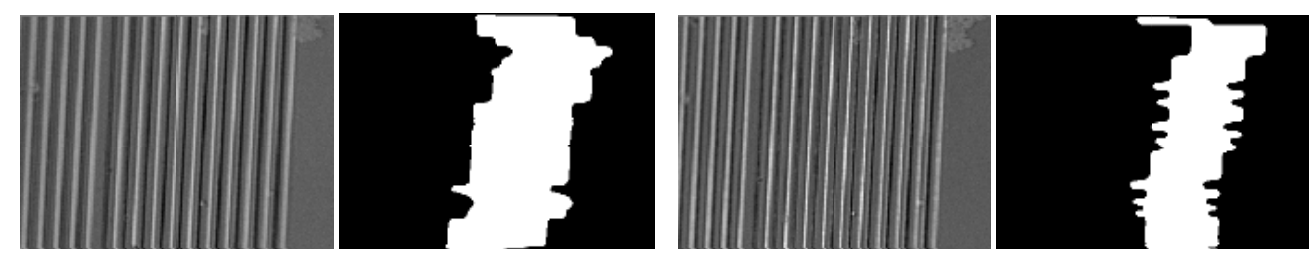

(c)
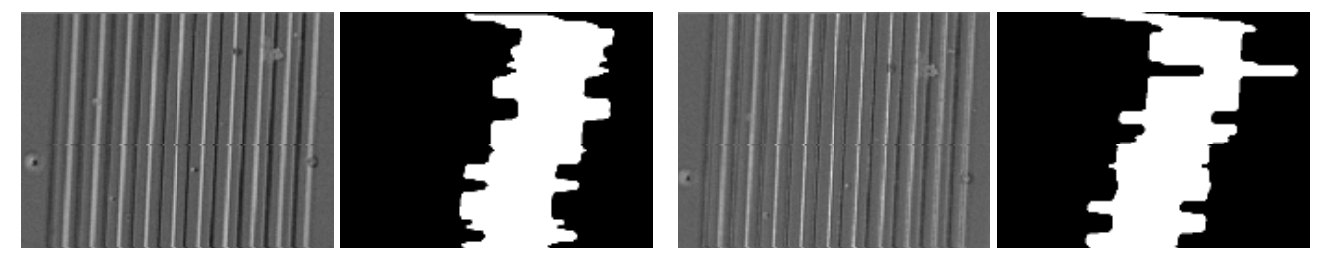

Figure 11 Shows the sectioning mask 


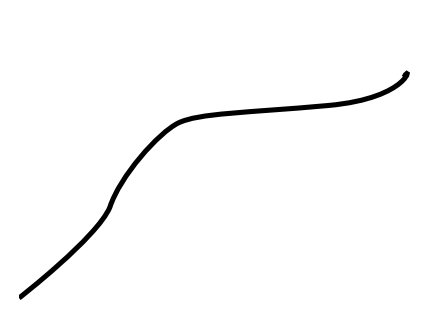

(a)

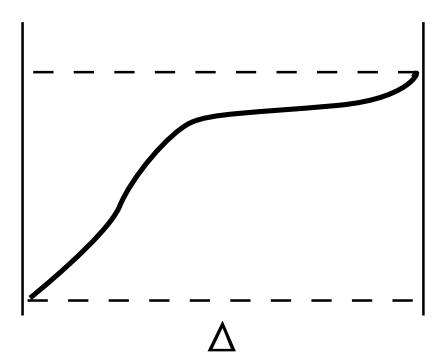

Intensity

(b)

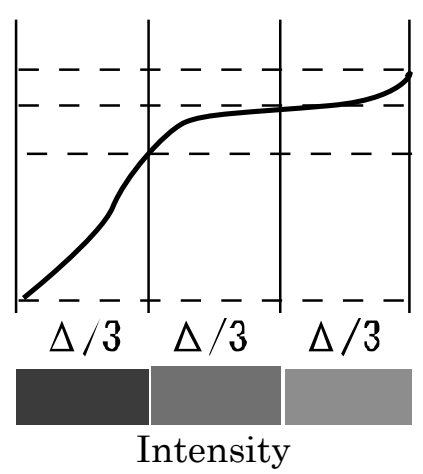

(C)

Figure 12 Relation between shear and the intensity. 